\title{
On certain inequalities associated to curvature properties of the nonlinear PPH reconstruction operator
}

\section{J.C. Trillo ${ }^{1 *}$ (D) and P. Ortiz}

“Correspondence: jc.trillo@upct.es 'Departamento de Matemática Aplicada y Estadística, Universidad Politécnica de Cartagena,

Cartagena, Spain

\section{Springer}

\begin{abstract}
In this paper we study the curvature term for the Lagrange and PPH (Amat et al. in Found. Comput. Math. 6:193-225, 2006 and Ortiz and Trillo in Preprint, arXiv:1811.10566, 2018) reconstruction operators in uniform and nonuniform meshes. We also make a comparison between both curvature terms in order to obtain an inequality which clearly shows that the PPH reconstruction presents a lower curvature term. Presenting a low curvature term is crucial in some applications, such as smoothing splines.
\end{abstract}

MSC: 41A05; 41A10; 65D17

Keywords: Interpolation; Approximation; Curvature; Reconstruction operator; Nonlinearity; Nonuniform grids

\section{Introduction}

Reconstruction and subdivision operators have been studied, analyzed and implemented in computer aided geometric design, giving rise to interesting applications in different fields of science. Subdivision schemes provide easy and fast algorithms for the generation of curves and surfaces from a coarse initial set of control points. They are closely related to reconstruction operators.

Starting from a given set of data, the target of the reconstruction operators is to obtain a piecewise function $p(x)$ which interpolates or approximates the data, preserving certain properties which are of interest because of some geometrical or physical reasons. One particular case is given by smoothing splines (see $[3,7])$ in a given interval $[a, b]$. They are built through polynomial reconstruction pieces that are connected in a smooth way at the control knots and satisfy the minimization problem

$$
\min _{p \in \prod_{n}} J(p):=\min _{p \in \prod_{n}} \int_{a}^{b} p^{\prime \prime}(x)^{2} d x+\sum_{j} \mu_{j}\left(p\left(x_{j}\right)-f_{j}\right)^{2}
$$

where $\prod_{n}$ stands for the set of polynomials of degree less or equal to $n$. The considered functional implies a balance, dominated by the weights $\mu_{j}$, between a low curvature term and a small value of the accumulated distance to the initial set of data $\left(x_{j}, f_{j}\right)$.

(c) The Author(s) 2019. This article is distributed under the terms of the Creative Commons Attribution 4.0 International License (http://creativecommons.org/licenses/by/4.0/), which permits unrestricted use, distribution, and reproduction in any medium, provided you give appropriate credit to the original author(s) and the source, provide a link to the Creative Commons license, and indicate if changes were made. 
PPH reconstruction was firstly defined in [1], although as subdivision scheme was already introduced in [4]. Later the PPH reconstruction operator was extended to allow for the use of nonuniform meshes [6], which is needed to link this reconstruction with general splines. This reconstruction is inherently a nonlinear interpolatory technique that has some remarkable characteristics. We mention those that are attractive for our purposes. In particular, a fixed centered stencil is used to build each polynomial piece, fourth-order accuracy is reached in smooth convex regions, reduction to second-order occurs at the vicinity of singularities but the approximation order is not completely lost as it happens in the linear case, and Gibb's effect is avoided. Also we especially remark two more properties which are going to be crucial for this reconstruction: convexity preservation when dealing with initial discrete set of convex data [6] and a low curvature term. The latter property about the curvature is part of what is going to be proven in the following sections. More precisely, we study the curvature term of the functional (1) for the Lagrange and PPH reconstructions, in the uniform and nonuniform cases. Then, due to these suitable properties, we think that connecting the $\mathrm{PPH}$ reconstruction with smoothing splines could result in very interesting applications.

The paper is organized as follows: In Sect. 2, we analyze the curvature term for the Lagrange and PPH reconstruction on uniform meshes. In Sect. 3, we study the case of nonuniform meshes. Finally, in Sect. 4, we present some conclusions and future perspectives.

\section{Study of the curvature term in uniform meshes}

Let us consider the set of values $f_{j-1}, f_{j}, f_{j+1}, f_{j+2}$ corresponding to subsequent ordinates at the abscissas $x_{j-1}, x_{j}, x_{j+1}, x_{j+2}$ of a regular grid $X$ with fixed grid spacing $h=x_{j+1}-x_{j}$. The set of polynomials $p(x)$, which pass through the central points $\left(x_{j}, f_{j}\right)$ and $\left(x_{j+1}, f_{j+1}\right)$, can be written in terms of two free variables $A$ and $B$ as follows:

$$
\begin{aligned}
p(x):= & -\frac{1}{6}\left(x-x_{j}\right)\left(x_{j+1}-x\right)\left[A\left(1+\frac{x_{j+1}-x}{h}\right)+B\left(1+\frac{x-x_{j}}{h}\right)\right] \\
& +\frac{x_{j+1}-x}{h} f_{j}+\frac{x-x_{j}}{h} f_{j+1} .
\end{aligned}
$$

From now on, we will use the following definition of the local curvature term.

Definition 1 Given a polynomial $p(x)$ in an interval $\left[x_{j}, x_{j+1}\right]$, we define the local curvature term as

$$
C(p):=\int_{x_{j}}^{x_{j+1}} p^{\prime \prime}(x)^{2} d x
$$

In order to compute the local curvature term in Definition 1 for the set of polynomials given by (2), we proceed as follows. The difference between the evaluation of polynomial (2) and the corresponding initial data at the abscissas $x_{j-1}, x_{j+2}$ is given by

$$
p\left(x_{j-1}\right)-f_{j-1}=h^{2}\left(A-2 D_{j}\right), \quad p\left(x_{j+2}\right)-f_{j+2}=h^{2}\left(B-2 D_{j+1}\right),
$$

where $D_{j}$ and $D_{j+1}$ are the second-order divided differences

$$
D_{j}=\frac{f_{j-1}-2 f_{j}+f_{j+1}}{2 h^{2}}, \quad D_{j+1}=\frac{f_{j}-2 f_{j+1}+f_{j+2}}{2 h^{2}} .
$$


Computing the second derivative of the polynomial $p(x)$ in (2) and introducing this computation in the local curvature term of expression (1), we get

$$
C(p):=\int_{x_{j}}^{x_{j+1}} p^{\prime \prime}(x)^{2} d x=\frac{h}{3}\left(A^{2}+A B+B^{2}\right) .
$$

\subsection{Curvature term for the Lagrange reconstruction}

Let $p_{L}(x)$ be the third-degree Lagrange polynomial, which interpolates the data $\left(x_{j+s}, f_{j+s}\right)$, $s=-1,0,1,2$. In order to write $p_{L}(x)$ in the form of polynomial (2), we look for the appropriate values of the parameters $A$ and $B$, which allow for the remaining interpolation conditions to be satisfied:

$$
p_{L}\left(x_{j-1}\right)=f_{j-1}, \quad p_{L}\left(x_{j+2}\right)=f_{j+2} .
$$

Solving the last two equations, we get

$$
A_{L}=2 D_{j}, \quad B_{L}=2 D_{j+1} .
$$

Thus, the defined curvature term (6) takes the form

$$
C_{L}:=C\left(p_{L}\right)=\frac{4 h}{3}\left(D_{j}^{2}+D_{j} D_{j+1}+D_{j+1}^{2}\right)
$$

\subsection{Curvature term for the PPH reconstruction}

Let now $p_{H}(x)$ be the PPH polynomial (see [1]). This fourth-order reconstruction based also on the data $\left(x_{j+s}, f_{j+s}\right), s=-1,0,1,2$, basically proceeds as follows: firstly, a modification of either $f_{j-1}$ or $f_{j+2}$ is carried out in order to avoid the bad influence of a potential singularity at $\left[x_{j-1}, x_{j}\right]$ or $\left[x_{j+1}, x_{j+2}\right]$, respectively; secondly, a third-order Lagrange interpolation is applied to the modified data. Then, due to this intrinsically nonlinear nature, we need to consider two different cases to carry out the curvature study for $p_{H}(x)$. This is done in the following theorem.

Theorem 1 The curvature term associated with the PPH polynomial $p_{H}(x)$ in a uniform mesh with grid spacing $h$ is given by

$$
C_{H}= \begin{cases}\frac{4 h D_{j}^{2}\left(D_{j}^{2}-2 D_{j} D_{j+1}+13 D_{j+1}^{2}\right)}{3\left(D_{j}+D_{j+1}\right)^{2}} & \text { if }\left|D_{j}\right| \leq\left|D_{j+1}\right| \& D_{j} D_{j+1}>0, \\ \frac{4 h D_{j}^{2}}{3} & \text { if }\left|D_{j}\right| \leq\left|D_{j+1}\right| \& D_{j} D_{j+1} \leq 0, \\ \frac{4 h D_{j+1}^{2}\left(13 D_{j}^{2}-2 D_{j} D_{j+1}+D_{j+1}^{2}\right)}{3\left(D_{j}+D_{j+1}\right)^{2}} & \text { if }\left|D_{j}\right|>\left|D_{j+1}\right| \& D_{j} D_{j+1}>0, \\ \frac{4 h D_{j+1}^{2}}{3} & \text { if }\left|D_{j}\right|>\left|D_{j+1}\right| \& D_{j} D_{j+1} \leq 0,\end{cases}
$$

where $D_{j}$ and $D_{j+1}$ are the second-order divided differences defined as

$$
D_{j}=\frac{f_{j-1}-2 f_{j}+f_{j+1}}{2 h^{2}}, \quad D_{j+1}=\frac{f_{j}-2 f_{j+1}+f_{j+2}}{2 h^{2}} .
$$

Moreover, for all cases $C_{H}$ satisfies $C_{L}-C_{H} \geq 0$. 
Proof Depending on the absolute values of the second-order divided differences $D_{j}$ and $D_{j+1}$ in (5), we analyze the following two cases:

Case 1. $\left|D_{j}\right| \leq\left|D_{j+1}\right|$, i.e., a potential singularity lies at $\left[x_{j+1}, x_{j+2}\right]$.

In this case, in order to build the $p_{H}(x)$ in the form of polynomial (2), we need to impose the following two conditions:

$$
p_{H}\left(x_{j-1}\right)=f_{j-1}, \quad p_{H}\left(x_{j+2}\right)=\widetilde{f}_{j+2},
$$

where $\widetilde{f}_{j+2}$ represents the modified value at $x_{j+2}$, and it is computed by (see [1] for more details)

$$
\widetilde{f}_{j+2}=f_{j+2}-4 h^{2}\left(\frac{D_{j}+D_{j+1}}{2}-\widetilde{D}_{j}\right)
$$

where $\widetilde{D}_{j}$ stands for the extended harmonic mean defined by

$$
\widetilde{D}_{j}= \begin{cases}\frac{2 D_{j} D_{j+1}}{D_{j}+D_{j+1}} & \text { if } D_{j} D_{j+1}>0 \\ 0 & \text { else. }\end{cases}
$$

Solving equations (10) for the free parameters results in

$$
A_{H}=2 D_{j}, \quad B_{H}=4 \widetilde{D}_{j}-2 D_{j}
$$

Depending on the sign of the product $D_{j} D_{j+1}$, the parameter $B_{H}$ takes a different expression, and therefore the same happens for the curvature term $C_{H}$ defined by $C_{H}:=C\left(p_{H}\right)$, according to expression (6). We consider now the following new two cases:

Case 1.1. $D_{j} D_{j+1}>0$.

In this case the term $B_{H}$ reads

$$
B_{H}=\frac{2 D_{j}\left(3 D_{j+1}-D_{j}\right)}{D_{j}+D_{j+1}},
$$

and thus

$$
C_{H}=\frac{4 h D_{j}^{2}\left(D_{j}^{2}-2 D_{j} D_{j+1}+13 D_{j+1}^{2}\right)}{3\left(D_{j}+D_{j+1}\right)^{2}} .
$$

It is now interesting and, in fact, part of our objective with this computation to compare the obtained curvature with the previous result (8) for the usual third-order Lagrange polynomial. Performing this comparison, we reach to

$$
C_{L}-C_{H}=\frac{4 h D_{j+1}\left(D_{j+1}-D_{j}\right)^{2}\left(5 D_{j}+D_{j+1}\right)}{3\left(D_{j}+D_{j+1}\right)^{2}} \geq 0,
$$

which shows clearly that the curvature term $C_{H}$ for the $\mathrm{PPH}$ reconstruction is always lower than the corresponding curvature $C_{L}$ for the Lagrange polynomial. This could be an interesting property in practical applications related with manufacturing and graphical design.

We study now the other case. 
Case 1.2. $D_{j} D_{j+1} \leq 0$.

In this case the terms $B_{H}$ and $C_{H}$ read

$$
B_{H}=-2 D_{j}, \quad C_{H}=\frac{4 h D_{j}^{2}}{3} .
$$

Therefore the difference $C_{L}-C_{H}$ is now

$$
C_{L}-C_{H}=\frac{4 h D_{j+1}\left(D_{j}+D_{j+1}\right)}{3} \geq 0
$$

Again we see that also in this case the curvature term $C_{H}$ for the $\mathrm{PPH}$ reconstruction is lower than the corresponding curvature $C_{L}$ for the Lagrange polynomial.

Case 2. $\left|D_{j}\right|>\left|D_{j+1}\right|$, i.e., the potential singularity lies at $\left[x_{j-1}, x_{j}\right]$. In this second case, in order to build the polynomial $p_{H}(x)$ in the form (2), we need to impose the following two conditions:

$$
p_{H}\left(x_{j-1}\right)=\widetilde{f}_{j-1}, \quad p_{H}\left(x_{j+2}\right)=f_{j+2} \text {. }
$$

where $\widetilde{f}_{j-1}$ is the modified value at $x_{j-1}$. Its expression is given by (see [1] for more details)

$$
\widetilde{f}_{j-1}=f_{j-1}-4 h^{2}\left(\frac{D_{j}+D_{j+1}}{2}-\widetilde{D}_{j}\right) .
$$

Working in a similar way to Case 1 , we obtain

$$
A_{H}=4 \widetilde{D}_{j}-2 D_{j+1}, \quad B_{H}=2 D_{j+1},
$$

and, depending on the sign of the product $D_{j} D_{j+1}$, we consider two subcases:

Case 2.1. $D_{j} D_{j+1}>0$.

Replacing $\widetilde{D}_{j}$ by (12) in the expression of $A_{H}$, we get

$$
A_{H}=\frac{2 D_{j+1}\left(3 D_{j}-D_{j+1}\right)}{D_{j}+D_{j+1}},
$$

and therefore from (6) we have

$$
C_{H}=\frac{4 h D_{j+1}^{2}\left(13 D_{j}^{2}-2 D_{j} D_{j+1}+D_{j+1}^{2}\right)}{3\left(D_{j}+D_{j+1}\right)^{2}} .
$$

Computing the difference between the curvature terms $C_{L}$ and $C_{H}$, we obtain

$$
C_{L}-C_{H}=\frac{4 h D_{j}\left(D_{j}-D_{j+1}\right)^{2}\left(D_{j}+5 D_{j+1}\right)}{3\left(D_{j}+D_{j+1}\right)^{2}} \geq 0 .
$$

Case 2.2. $D_{j} D_{j+1} \leq 0$. Replacing $\widetilde{D}_{j}$ by (12) in the expression of $A_{H}$, we get now

$$
A_{H}=-2 D_{j+1},
$$


and therefore from (6) this time we have

$$
C_{H}=\frac{4 h D_{j+1}^{2}}{3}
$$

Finally, the difference between both curvature terms writes

$$
C_{L}-C_{H}=\frac{4 h D_{j}\left(D_{j}+D_{j+1}\right)}{3} \geq 0
$$

We have just seen that for data in uniform grids, the curvature term in equation (1) associated to PPH reconstruction operator remains below the value of the curvature associated to Lagrange operator.

\section{Study of the curvature term in nonuniform meshes}

Let us consider the set of points $f_{j-1}, f_{j}, f_{j+1}, f_{j+2}$ corresponding to subsequent ordinates at the abscissas $x_{j-1}, x_{j}, x_{j+1}, x_{j+2}$ of a nonuniform mesh $X$. Let be $h_{j}=x_{j}-x_{j-1}, h_{j+1}=x_{j+1}-x_{j}$, $h_{j+2}=x_{j+2}-x_{j+1}$. Similarly to the uniform case, polynomials $p(x)$, which pass through the central points $\left(x_{j}, f_{j}\right)$ and $\left(x_{j+1}, f_{j+1}\right)$, can be written as

$$
\begin{aligned}
p(x):= & -\frac{1}{6}\left(x-x_{j}\right)\left(x_{j+1}-x\right)\left[A\left(1+\frac{x_{j+1}-x}{h_{j+1}}\right)+B\left(1+\frac{x-x_{j}}{h_{j+1}}\right)\right] \\
& +\frac{x_{j+1}-x}{h_{j+1}} f_{j}+\frac{x-x_{j}}{h_{j+1}} f_{j+1} .
\end{aligned}
$$

At the boundary points $x_{j-1}, x_{j+2}$ of the interval, the distance of the polynomial to the initial data is:

$$
\begin{aligned}
p\left(x_{j-1}\right)-f_{j-1}= & \frac{h_{j}\left(h_{j}+h_{j+1}\right)}{6 h_{j+1}}\left(A\left(h_{j}+2 h_{j+1}\right)+B\left(h_{j+1}-h_{j}\right)-6 D_{j} h_{j+1}\right), \\
p\left(x_{j+2}\right)-f_{j+2}= & \frac{h_{j+2}\left(h_{j+1}+h_{j+2}\right)}{6 h_{j+1}}\left(A\left(h_{j+1}-h_{j+2}\right)+B\left(2 h_{j+1}+h_{j+2}\right)\right. \\
& \left.-6 D_{j+1} h_{j+1}\right),
\end{aligned}
$$

where $D_{j}$ and $D_{j+1}$ are the general divided differences defined by

$$
\begin{aligned}
& D_{j}=\frac{f_{j-1}}{h_{j}\left(h_{j}+h_{j+1}\right)}-\frac{f_{j}}{h_{j} h_{j+1}}+\frac{f_{j+1}}{h_{j+1}\left(h_{j}+h_{j+1}\right)}, \\
& D_{j+1}=\frac{f_{j}}{h_{j+1}\left(h_{j+1}+h_{j+2}\right)}-\frac{f_{j+1}}{h_{j+1} h_{j+2}}+\frac{f_{j+2}}{h_{j+2}\left(h_{j+1}+h_{j+2}\right)} .
\end{aligned}
$$

Introducing the second derivative of (19) in the curvature term of (1), we get

$$
C(p)=\int_{x_{j}}^{x_{j+1}} p^{\prime \prime}(x)^{2} d x=\frac{h_{j+1}}{3}\left(A^{2}+A B+B^{2}\right)=\frac{h_{j+1}}{3}\left((A+B)^{2}-A B\right) .
$$




\subsection{Curvature term for the Lagrange reconstruction in nonuniform meshes}

When $p(x)$ is the Lagrange polynomial $p_{L}(x)$, it verifies

$$
p_{L}\left(x_{j-1}\right)=f_{j-1}, \quad p_{L}\left(x_{j+2}\right)=f_{j+2} \text {. }
$$

Previous conditions and Eq. (20) result in the following linear system for $A$ and $B$ :

$$
\begin{aligned}
& A_{L}\left(h_{j}+2 h_{j+1}\right)+B_{L}\left(h_{j+1}-h_{j}\right)=6 D_{j} h_{j+1}, \\
& A_{L}\left(h_{j+1}-h_{j+2}\right)+B_{L}\left(2 h_{j+1}+h_{j+2}\right)=6 D_{j+1} h_{j+1} .
\end{aligned}
$$

Solving this system, we obtain the parameters $A$ and $B$ for the Lagrange polynomial as

$$
\begin{aligned}
& A_{L}=\frac{2\left[D_{j}\left(2 h_{j+1}+h_{j+2}\right)+D_{j+1}\left(h_{j}-h_{j+1}\right)\right]}{h_{j}+h_{j+1}+h_{j+2}}, \\
& B_{L}=\frac{2\left[D_{j}\left(h_{j+2}-h_{j+1}\right)+D_{j+1}\left(h_{j}+2 h_{j+1}\right)\right]}{h_{j}+h_{j+1}+h_{j+2}} .
\end{aligned}
$$

It is convenient to observe that

$$
\begin{aligned}
& A_{L}+B_{L}=4 M_{j}, \\
& A_{L}-B_{L}=\frac{6 h_{j+1}\left(D_{j}-D_{j+1}\right)}{h_{j}+h_{j+1}+h_{j+2}} .
\end{aligned}
$$

Plugging these values into expression (22), we get the curvature term $C_{L}=C\left(p_{L}\right)$ for the Lagrange reconstruction.

\subsection{Curvature term for the PPH reconstruction in nonuniform meshes}

The PPH reconstruction in nonuniform meshes is defined in the interval $\left[x_{j}, x_{j+1}\right]$ by using the data $f_{j-1}, f_{j}, f_{j+1}, f_{j+2}$ at the abscissas $x_{j-1}, x_{j}, x_{j+1}, x_{j+2}$ in the following way: depending on the relative size of $\left|D_{j}\right|$ an $\left|D_{j+1}\right|$, we substitute either $f_{j-1}$ for $\tilde{f}_{j-1}$ or $f_{j+2}$ for $\tilde{f}_{j+2}$. After this replacement, Lagrange reconstruction is applied to the new set of data. We remark that the initial substitution is made in order to adapt to the presence of potential singularities and, at the same time, maintain the fourth-order accuracy of Lagrange reconstruction in smooth convex areas.

In what follows, we present some expressions that we will need to derive the curvature term. For more information about these expressions, see [6]. The mentioned substitutions, depending on the relative size of $\left|D_{j}\right|$ and $\left|D_{j+1}\right|$, take the form

$$
\begin{aligned}
& \widetilde{f}_{j-1}=f_{j-1}-\frac{h_{j}\left(h_{j}+h_{j+1}\right)}{w_{j}}\left(M_{j}-\widetilde{V}_{j}\right), \\
& \widetilde{f}_{j+2}=f_{j+2}-\frac{h_{j+2}\left(h_{j+1}+h_{j+2}\right)}{w_{j+1}}\left(M_{j}-\widetilde{V}_{j}\right),
\end{aligned}
$$

where $M_{j}$ and $\widetilde{V}_{j}$ are weighted arithmetic and harmonic means of $D_{j}$ and $D_{j+1}$, that is,

$$
M_{j}=w_{j} D_{j}+w_{j+1} D_{j+1},
$$




$$
\widetilde{V}_{j}= \begin{cases}\frac{D_{j} D_{j+1}}{w_{j} D_{j+1}+w_{j+1} D_{j}} & \text { if } D_{j} D_{j+1}>0, \\ 0 & \text { otherwise }\end{cases}
$$

and the weights $w_{j}, w_{j+1}$ are defined by

$$
\begin{aligned}
& w_{j}=\frac{h_{j+1}+2 h_{j+2}}{2\left(h_{j}+h_{j+1}+h_{j+2}\right)}, \\
& w_{j+1}=\frac{h_{j+1}+2 h_{j}}{2\left(h_{j}+h_{j+1}+h_{j+2}\right)}=1-w_{j} .
\end{aligned}
$$

From Eqs. (28) and (29) we obtain

$$
M_{j}-\widetilde{V}_{j}= \begin{cases}\frac{w_{j} w_{j+1}\left(D_{j+1}-D_{j}\right)^{2}}{w_{j} D_{j+1}+w_{j+1} D_{j}} & \text { if } D_{j} D_{j+1}>0 \\ M_{j} & \text { otherwise. }\end{cases}
$$

This expression will be used later.

The divided differences $\widetilde{D}_{j}$ and $\widetilde{D}_{j+1}$ calculated with the PPH ordinates $\widetilde{f}_{j-1}$ and $\widetilde{f}_{j+2}$ are now given by

$$
\begin{aligned}
& \widetilde{D}_{j}=\frac{\widetilde{f}_{j-1}}{h_{j}\left(h_{j}+h_{j+1}\right)}-\frac{f_{j}}{h_{j} h_{j+1}}+\frac{f_{j+1}}{h_{j+1}\left(h_{j}+h_{j+1}\right)}, \\
& \widetilde{D}_{j+1}=\frac{f_{j}}{h_{j+1}\left(h_{j+1}+h_{j+2}\right)}-\frac{f_{j+1}}{h_{j+1} h_{j+2}}+\frac{\widetilde{f}_{j+2}}{h_{j+2}\left(h_{j+1}+h_{j+2}\right)},
\end{aligned}
$$

and their difference with (21) becomes

$$
\begin{aligned}
& D_{j}-\widetilde{D}_{j}=\frac{f_{j-1}-\widetilde{f}_{j-1}}{h_{j}\left(h_{j}+h_{j+1}\right)}=\frac{M_{j}-\widetilde{V}_{j}}{w_{j}}, \\
& D_{j+1}-\widetilde{D}_{j+1}=\frac{f_{j+2}-\widetilde{f}_{j+2}}{h_{j+2}\left(h_{j+1}+h_{j+2}\right)}=\frac{M_{j}-\widetilde{V}_{j}}{w_{j+1}} .
\end{aligned}
$$

We are now ready to compute the curvature term associated to $\mathrm{PPH}$ reconstruction $p(x)=$ $p_{H}(x)$.

Theorem 2 The curvature term associated to the PPH polynomial $p_{H}(x)$ in a nonuniform mesh satisfies

(1.1) If $\left|D_{j}\right| \leq\left|D_{j+1}\right| \& D_{j} D_{j+1}>0$,

$$
\begin{aligned}
C_{L}-C_{H}= & \frac{12 h_{j+1}^{3} w_{j} w_{j+1}^{2}}{\left(2 h_{j}+h_{j+1}\right)^{2}}\left(\frac{w_{j} D_{j+1}+w_{j+1} D_{j}+D_{j}}{\left(w_{j} D_{j+1}+w_{j+1} D_{j}\right)^{2}}\right)\left(D_{j+1}-D_{j}\right)^{3} \\
& +4 h_{j+1}\left(M_{j}^{2}-\widetilde{V}_{j}^{2}\right) . \\
C_{L}-C_{H} \geq 0 . &
\end{aligned}
$$

(1.2) If $\left|D_{j}\right| \leq\left|D_{j+1}\right| \& D_{j} D_{j+1} \leq 0$,

$$
C_{L}-C_{H}=\frac{8 M_{j} h_{j+1}}{\left(2 h_{j}+h_{j+1}\right)^{2}}\left(2\left(h_{j}^{2}+h_{j} h_{j+1}+h_{j+1}^{2}\right) M_{j}-3 h_{j+1}^{2} D_{j}\right)
$$


$C_{L}-C_{H} \geq 0$, under one of these natural conditions:

(1.2.1) If $M_{j}$ and $D_{j}$ have different sign.

(1.2.2) If $M_{j}$ and $D_{j}$ have the same sign and

$$
\frac{M_{j}}{D_{j}}>\frac{3 h_{j+1}^{2}}{2\left(h_{j}^{2}+h_{j} h_{j+1}+h_{j+1}^{2}\right)} .
$$

(2.1) If $\left|D_{j}\right|>\left|D_{j+1}\right| \& D_{j} D_{j+1}>0$,

$$
\begin{aligned}
C_{L}-C_{H}= & \frac{12 h_{j+1}^{3} w_{j}^{2} w_{j+1}}{\left(h_{j+1}+2 h_{j+2}\right)^{2}}\left(\frac{w_{j} D_{j+1}+w_{j+1} D_{j}+D_{j+1}}{\left(w_{j} D_{j+1}+w_{j+1} D_{j}\right)^{2}}\right)\left(D_{j}-D_{j+1}\right)^{3} \\
& +4 h_{j+1}\left(M_{j}^{2}-\widetilde{V}_{j}^{2}\right)
\end{aligned}
$$

$C_{L}-C_{H} \geq 0$.

(2.2) If $\left|D_{j}\right|>\left|D_{j+1}\right| \& D_{j} D_{j+1} \leq 0$,

$$
C_{L}-C_{H}=\frac{8 M_{j} h_{j+1}}{\left(h_{j+1}+2 h_{j+2}\right)^{2}}\left(2\left(h_{j+1}^{2}+h_{j+1} h_{j+2}+h_{j+2}^{2}\right) M_{j}-3 h_{j+1}^{2} D_{j+1}\right),
$$

$C_{L}-C_{H} \geq 0$, under one of these natural conditions:

(2.2.1) If $M_{j}$ and $D_{j+1}$ have different sign.

(2.2.2) If $M_{j}$ and $D_{j+1}$ have the same sign and

$$
\frac{M_{j}}{D_{j+1}}>\frac{3 h_{j+1}^{2}}{2\left(h_{j+1}^{2}+h_{j+1} h_{j+2}+h_{j+2}^{2}\right)} .
$$

Proof We need to consider two main cases.

Case 1. $\left|D_{j}\right| \leq\left|D_{j+1}\right|$, i.e., the possible singularity is at $\left[x_{j+1}, x_{j+2}\right]$ and

$$
p_{H}\left(x_{j-1}\right)=f_{j-1}, \quad p_{H}\left(x_{j+2}\right)=\widetilde{f}_{j+2} .
$$

From previous conditions and Eqs. (20) and (27b), we get the following linear system in $A$ and $B$ :

$$
\begin{aligned}
& A_{H}\left(h_{j}+2 h_{j+1}\right)+B_{H}\left(h_{j+1}-h_{j}\right)=6 D_{j} h_{j+1}, \\
& A_{H}\left(h_{j+1}-h_{j+2}\right)+B_{H}\left(2 h_{j+1}+h_{j+2}\right)=6 \widetilde{D}_{j+1} h_{j+1} .
\end{aligned}
$$

We observe that this system has the same form as the system for the Lagrange case (24), except for $\widetilde{D}_{j+1}$. Its solution is

$$
\begin{aligned}
& A_{H}=\frac{2\left[D_{j}\left(2 h_{j+1}+h_{j+2}\right)+\widetilde{D}_{j+1}\left(h_{j}-h_{j+1}\right)\right]}{h_{j}+h_{j+1}+h_{j+2}}, \\
& B_{H}=\frac{2\left[D_{j}\left(h_{j+2}-h_{j+1}\right)+\widetilde{D}_{j+1}\left(h_{j}+2 h_{j+1}\right)\right]}{h_{j}+h_{j+1}+h_{j+2}},
\end{aligned}
$$


which can also be expressed as

$$
\begin{aligned}
& A_{H}=\frac{6 D_{j} h_{j+1}+4\left(h_{j}-h_{j+1}\right) \widetilde{V}_{j}}{2 h_{j}+h_{j+1}}, \\
& B_{H}=\frac{-6 D_{j} h_{j+1}+4\left(h_{j}+2 h_{j+1}\right) \widetilde{V}_{j}}{2 h_{j}+h_{j+1}} .
\end{aligned}
$$

From (36) we can easily see that

$$
A_{H}+B_{H}=4 \widetilde{V}_{j}
$$

We also point out that parameters $A_{L}, B_{L}$ and $A_{H}, B_{H}$ are related by

$$
\begin{aligned}
& A_{H}=A_{L}-\left(2-\frac{6 h_{j+1}}{2 h_{j}+h_{j+1}}\right)\left(M_{j}-\widetilde{V}_{j}\right), \\
& B_{H}=B_{L}-\left(2+\frac{6 h_{j+1}}{2 h_{j}+h_{j+1}}\right)\left(M_{j}-\widetilde{V}_{j}\right) .
\end{aligned}
$$

Taking into account Eqs. (22), (26), (37) and (38), we obtain the difference $C_{L}-C_{H}$ between Lagrange and PPH curvature terms as

$$
\begin{aligned}
C_{L}-C_{H}= & \frac{12 h_{j+1}^{3}\left(M_{j}-\tilde{V}_{j}\right)}{\left(2 h_{j}+h_{j+1}\right)^{2}}\left(2 w_{j+1}\left(D_{j+1}-D_{j}\right)-\left(M_{j}-\widetilde{V}_{j}\right)\right) \\
& +4 h_{j+1}\left(M_{j}^{2}-\widetilde{V}_{j}^{2}\right) .
\end{aligned}
$$

Introducing the expression (31) of the difference $M_{j}-\widetilde{V}_{j}$ in previous equation, the following subcases appear:

Case 1.1. $D_{j} D_{j+1}>0$. Then

$$
\begin{aligned}
C_{L}-C_{H}= & \frac{12 h_{j+1}^{3} w_{j} w_{j+1}^{2}}{\left(2 h_{j}+h_{j+1}\right)^{2}}\left(\frac{w_{j} D_{j+1}+w_{j+1} D_{j}+D_{j}}{\left(w_{j} D_{j+1}+w_{j+1} D_{j}\right)^{2}}\right)\left(D_{j+1}-D_{j}\right)^{3} \\
& +4 h_{j+1}\left(M_{j}^{2}-\widetilde{V}_{j}^{2}\right) .
\end{aligned}
$$

On the one hand, as the sign of $D_{j}$ equals to the sign of $D_{j+1}$, we get $M_{j}^{2} \geq \widetilde{V}_{j}^{2}$.

On the other hand, since we are in the case $\left|D_{j}\right| \leq\left|D_{j+1}\right|$, this implies that

$$
\left(w_{j} D_{j+1}+w_{j+1} D_{j}+D_{j}\right)\left(D_{j+1}-D_{j}\right)^{3} \geq 0 .
$$

Thus, $C_{L}-C_{H} \geq 0$.

Case 1.2. $D_{j} D_{j+1} \leq 0$. This time

$$
C_{L}-C_{H}=\frac{8 M_{j} h_{j+1}}{\left(2 h_{j}+h_{j+1}\right)^{2}}\left(2\left(h_{j}^{2}+h_{j} h_{j+1}+h_{j+1}^{2}\right) M_{j}-3 h_{j+1}^{2} D_{j}\right) .
$$

So $C_{L}-C_{H}$ will be positive if $M_{j}$ and $2\left(h_{j}^{2}+h_{j} h_{j+1}+h_{j+1}^{2}\right) M_{j}-3 h_{j+1}^{2} D_{j}$ have the same sign. This happens in the following cases: 
Case 1.2.1. $M_{j}$ and $D_{j}$ (the lower divided difference in absolute value) have different sign.

Case 1.2.2. $M_{j}$ and $D_{j}$ have the same sign and $\frac{M_{j}}{D_{j}}>\frac{3 h_{j+1}^{2}}{2\left(h_{j}^{2}+h_{j} h_{j+1}+h_{j+1}^{2}\right)}$.

The latter conditions are not always satisfied. However, we can solve this situation by paying proper attention to the following facts:

- Case 1.2 will only take place around inflection points on the underlying function.

Therefore, if we work with data corresponding to strictly convex or concave functions this case will never happen.

- Case 1.2.2 will not occur around discontinuities except for extremely nonuniform grids where $w_{j} \approx 1$, since $M_{j}$ and $D_{j}$ have the same sign if and only if $\left|\frac{D_{j+1}}{D_{j}}\right|<\frac{w_{j}}{w_{j+1}}$.

- Under the assumption that for the given data condition in Case 1.2.1 is not satisfied, although this is a rare situation, we can consider the replacement at this concrete interval of the original data $f_{j-1}$ by $\tilde{f}_{j-1}$ according to (27a) instead of $f_{j+2}$ by $\tilde{f}_{j+2}$ in order to attain $C_{L} \geq C_{H}$. This observation is easily proven because we go directly to Case 2.2.1. Thus, we give priority to the minimization of the curvature instead to the adaption to possible singularities. Notice that as mentioned in the previous point, there should not be a singularity at the considered interval but for exceptional cases.

Case 2. $\left|D_{j}\right|>\left|D_{j+1}\right|$, i.e., the possible singularity is at $\left[x_{j-1}, x_{j}\right]$. Here

$$
p_{H}\left(x_{j-1}\right)=\widetilde{f}_{j-1}, \quad p_{H}\left(x_{j+2}\right)=f_{j+2} .
$$

Previous conditions, together with Eqs. (20) and (27a), give the following linear system for $A$ and $B$ :

$$
\begin{aligned}
& A_{H}\left(h_{j}+2 h_{j+1}\right)+B_{H}\left(h_{j+1}-h_{j}\right)=6 \widetilde{D}_{j} h_{j+1}, \\
& A_{H}\left(h_{j+1}-h_{j+2}\right)+B_{H}\left(2 h_{j+1}+h_{j+2}\right)=6 D_{j+1} h_{j+1} .
\end{aligned}
$$

Its solution is

$$
\begin{aligned}
& A_{H}=\frac{2\left[\widetilde{D}_{j}\left(2 h_{j+1}+h_{j+2}\right)+D_{j+1}\left(h_{j}-h_{j+1}\right)\right]}{h_{j}+h_{j+1}+h_{j+2}}, \\
& B_{H}=\frac{2\left[\widetilde{D}_{j}\left(h_{j+2}-h_{j+1}\right)+D_{j+1}\left(h_{j}+2 h_{j+1}\right)\right]}{h_{j}+h_{j+1}+h_{j+2}},
\end{aligned}
$$

which can also be expressed as

$$
\begin{gathered}
A_{H}=\frac{-6 D_{j+1} h_{j+1}+4\left(2 h_{j+1}+h_{j+2}\right) \widetilde{V}_{j}}{h_{j+1}+2 h_{j+2}}, \\
B_{H}=\frac{6 D_{j+1} h_{j+1}+4\left(h_{j+2}-h_{j+1}\right) \widetilde{V}_{j}}{h_{j+1}+2 h_{j+2}},
\end{gathered}
$$

where we see that, as in Case 1,

$$
A_{H}+B_{H}=4 \widetilde{V}_{j} .
$$


We also point out that parameters $A_{L}, B_{L}$ and $A_{H}, B_{H}$ are related by

$$
\begin{aligned}
& A_{H}=A_{L}-\left(2+\frac{6 h_{j+1}}{h_{j+1}+2 h_{j+2}}\right)\left(M_{j}-\widetilde{V}_{j}\right), \\
& B_{H}=B_{L}-\left(2-\frac{6 h_{j+1}}{h_{j+1}+2 h_{j+2}}\right)\left(M_{j}-\widetilde{V}_{j}\right) .
\end{aligned}
$$

Taking into account Eqs. (22), (26), (37) and (46), we also reach in this case to the expression for the difference $C_{L}-C_{H}$, between Lagrange and PPH curvature terms, given by

$$
\begin{aligned}
C_{L}-C_{H}= & \frac{12 h_{j+1}^{3}\left(M_{j}-\widetilde{V}_{j}\right)}{\left(h_{j+1}+2 h_{j+2}\right)^{2}}\left(2 w_{j}\left(D_{j}-D_{j+1}\right)-\left(M_{j}-\widetilde{V}_{j}\right)\right) \\
& +4 h_{j+1}\left(M_{j}^{2}-\widetilde{V}_{j}^{2}\right) .
\end{aligned}
$$

Using expression (31) of the difference $M_{j}-\widetilde{V}_{j}$ in previous equation, we get the subcases:

Case 2.1. $D_{j} D_{j+1}>0$. Here

$$
\begin{aligned}
C_{L}-C_{H}= & \frac{12 h_{j+1}^{3} w_{j}^{2} w_{j+1}}{\left(h_{j+1}+2 h_{j+2}\right)^{2}}\left(\frac{w_{j} D_{j+1}+w_{j+1} D_{j}+D_{j+1}}{\left(w_{j} D_{j+1}+w_{j+1} D_{j}\right)^{2}}\right)\left(D_{j}-D_{j+1}\right)^{3} . \\
& +4 h_{j+1}\left(M_{j}^{2}-\widetilde{V}_{j}^{2}\right) .
\end{aligned}
$$

On the one hand, $D_{j} D_{j+1}>0$ implies $M_{j}^{2} \geq \widetilde{V}_{j}^{2}$.

On the other hand, since $\left|D_{j}\right|>\left|D_{j+1}\right|$, we get

$$
\left(w_{j} D_{j+1}+w_{j+1} D_{j}+D_{j+1}\right)\left(D_{j}-D_{j+1}\right)^{3} \geq 0 .
$$

Thus, $C_{L}-C_{H} \geq 0$.

Case 2.2. $D_{j} D_{j+1} \leq 0$. Now

$$
C_{L}-C_{H}=\frac{8 M_{j} h_{j+1}}{\left(h_{j+1}+2 h_{j+2}\right)^{2}}\left(2\left(h_{j+1}^{2}+h_{j+1} h_{j+2}+h_{j+2}^{2}\right) M_{j}-3 h_{j+1}^{2} D_{j+1}\right) .
$$

So $C_{L}-C_{H}$ will be positive if $2\left(h_{j+1}^{2}+h_{j+1} h_{j+2}+h_{j+2}^{2}\right) M_{j}-3 h_{j+1}^{2} D_{j+1}$ has the same sign as $M_{j}$. This occurs when

Case 2.2.1. $M_{j}$ and $D_{j+1}$ (the lower divided difference in absolute value) have different sign.

Case 2.2.2. $M_{j}$ and $D_{j+1}$ have the same sign and $\frac{M_{j}}{D_{j+1}}>\frac{3 h_{j+1}^{2}}{2\left(h_{j+1}^{2}+h_{j+1} h_{j+2}+h_{j+2}^{2}\right)}$.

At this point, the same observations as in Case 1.2 can be stated. That is, the latter conditions are not always satisfied. However, we can solve this situation by paying proper attention to the following facts:

- Case 2.2 will only appear around inflection points. Therefore, the case is avoided if we consider only data corresponding to strictly convex or concave functions.

- Case 2.2.2 will not occur around discontinuities except for extremely nonuniform grids where $w_{j+1} \approx 1$, since $M_{j}$ and $D_{j+1}$ have the same sign if and only if $\left|\frac{D_{j}}{D_{j}+1}\right|<\frac{w_{j+1}}{w_{j}}$. 
- Under the assumption that for the given data condition in Case 2.2.1 is not satisfied, albeit this is not a common situation, we can give priority, as it happened in Case 1.2.2, to the minimization of the curvature instead to the adaption to possible singularities. Then, we consider in this case the replacement at this particular interval of the original data $f_{j+2}$ by $\tilde{f}_{j+2}$ according to $(27 \mathrm{~b})$ instead of $f_{j-1}$ by $\tilde{f}_{j-1}$ in order to attain $C_{L} \geq C_{H}$. Again this observation is trivial to prove.

\section{Conclusions and perspectives}

We have obtained some inequalities which demonstrate that PPH reconstruction operator behaves better than usual linear Lagrange reconstruction operator regarding curvature issues. This study complements other previous results $[1,2,6]$ where it was proven that PPH reconstruction preserves also the convexity properties of the initial data. This property is also inherited by the associated subdivision scheme $[5,8]$.

This opens up a potential future work connecting PPH reconstruction with smoothing splines in order to obtain a PPH-type reconstruction of class $C^{2}$ in the whole interval with interesting convexity-preserving properties and low curvature term. Notice that piecewise $\mathrm{PPH}$ reconstruction is only continuous at the joint nodes.

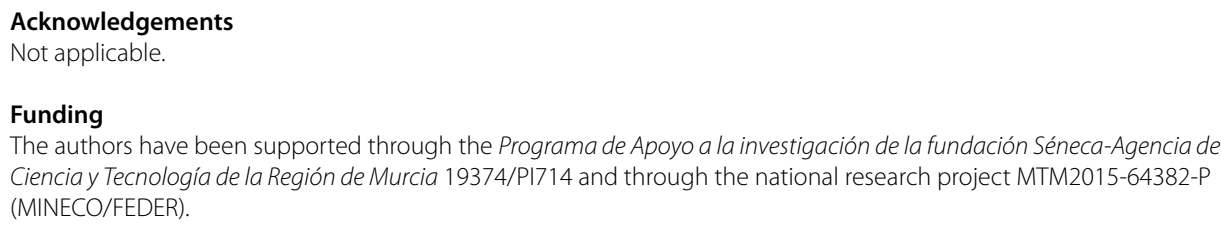

Availability of data and materials

Not applicable.

Competing interests

The authors declare that they have no competing interests.

Authors' contributions

Both two authors worked together, prepared, read and approved the manuscript.

\section{Publisher's Note}

Springer Nature remains neutral with regard to jurisdictional claims in published maps and institutional affiliations.

Received: 9 August 2018 Accepted: 6 January 2019 Published online: 14 January 2019

\section{References}

1. Amat, S., Donat, R., Liandrat, J., Trillo, J.C.: Analysis of a new nonlinear subdivision scheme. Applications in image processing. Found. Comput. Math. 6(2), 193-225 (2006)

2. Amat, S., Donat, R., Trillo, J.C.: Proving convexity preserving properties of interpolatory subdivision schemes through reconstruction operators. Appl. Math. Comput. 219(14), 7413-7421 (2013)

3. de Boor, C.: A Practical Guide to Splines. Springer, New York (1978)

4. Floater, M.S., Michelli, C.A.: Nonlinear stationary subdivision. In: Govil, N.K., Mohapatra, N., Nashed, Z., Sharma, A., Szabados, J. (eds.) Approximation Theory: In Memory of A.K. Varna, pp. 209-224 (1998)

5. Kuijt, F., van Damme, R.: Convexity preserving interpolatory subdivision schemes. Constr. Approx. 14, 609-630 (1998)

6. Ortiz, P., Trillo, J.C.: A Nonlinear interpolatory reconstruction operator on nonuniform grids (2018). arXiv:1811.10566. Preprint

7. Reinsch, C.H.: Smoothing by spline functions. Numer. Math. 10, 177-183 (1967)

8. Trillo, J.C.: Nonlinear multiresolution and applications in image processing. PhD Thesis, University of Valencia, Spain (2007) 